\title{
A opinião de estudantes de medicina e enfermagem sobre gravidez na adolescência
}

\author{
The view of medical and nursing students on adolescent pregnancy
}

\author{
Márcia Elisa Jager ${ }^{[a]}$, Danielle da Costa Souto ${ }^{[b]}$, Raquel Flores de Lima ${ }^{[c]}$, \\ Meiridiane Domingues de Deus ${ }^{[\mathrm{d}]}$, Ana Cristina Garcia Dias[e]
}

\footnotetext{
${ }^{[a]}$ Mestre em Psicologia pela Universidade Federal de Santa Maria, Santa Maria, RS - Brasil e-mail: marciajager@yahoo. com.br

${ }^{[b]}$ Mestranda em Psicologia pela Universidade Federal de Santa Maria, Santa Maria, RS - Brasil, e-mail: daniellessouto@gmail.com

${ }^{[c]}$ Mestranda em Psicologia pela Universidade Federal de Santa Maria, Santa Maria, RS - Brasil, e-mail: quel_fl@yahoo.com.br

${ }^{[\mathrm{d}]}$ Mestranda em Psicologia pela Universidade Federal de Santa Maria, Santa Maria, RS - Brasil, e-mail:meiridddeus@gmail.com

${ }^{[\mathrm{e}]}$ Mestranda em Psicologia pela Universidade Federal de Santa Maria, Santa Maria, RS - Brasil, e-mail: anacristinagarciadias@ gmail.com
}

Recebido: 17/07/2013 Received: $07 / 17 / 2013$

Aprovado: 08/10/2013 Approved: 10/08/2013

\section{Resumo}

Este estudo busca conhecer as opiniões de estudantes de medicina e enfermagem sobre as causas da gravidez durante a adolescência e sobre o atendimento em saúde prestado a essa população. Participaram deste estudo 106 estudantes universitários de ambos os sexos, com idades entre 18 e 27 anos, matriculados nos semestre iniciais dos cursos de Enfermagem e Medicina da Universidade de Passo Fundo. Os principais resultados indicam que os estudantes reconhecem um grau de complexidade envolvido no fenômeno de gravidez adolescente e que seu atendimento necessita ser multidisciplinar, uma vez que envolve tanto aspectos biológicos e sociológicos quanto aspectos culturais e psicológicos. Entretanto, os participantes deste estudo não reconhecem, de fato, a influência do contexto sociocultural sobre a experiência da gestação e maternidade adolescente, apresentando crenças estereotipadas em relação a estas experiências.

Palavras-Chave: Estudantes. Medicina. Enfermagem. Gravidez na adolescência. Adolescente.

\begin{abstract}
This study seeks to understand the opinions of medical and nursing students about the causes of teenage pregnancy and the health care provided to this population. The study included 106 college students of both sexes, aged between 18 and 27 years, enrolled in the initial half of the Nursing and Medicine courses at the University of Passo Fundo. The main results indicated that students acknowledge a degree of complexity involved in the phenomenon of teenage pregnancy and that their care needs be multidisciplinary, since it involves biological, sociological and cultural aspects as much as psychological aspects. However, the study participants did not recognize, in fact, the influence of the sociocultural context in the experience of pregnancy and teen motherhood, showing stereotypical beliefs regarding these experiences.
\end{abstract}

Keywords: Students. Medicine. Nursing. Teenage pregnancy. Teenager.

Psicol. Argum. 2014, 32(79), Supl. 2, 77-88 


\section{Introdução}

0 trabalho com adolescentes exige conhecimentos teóricos e técnicos específicos, aliados ao interesse pelo comportamento humano e sensibilidade para perceber as influências de questões culturais e socioeconômicas no comportamento dos jovens. É necessário gostar de adolescentes, ter empatia por eles e tolerar suas demandas e frustrações que o trabalho com pacientes nesta faixa etária pode gerar no profissional. Caso o profissional não mostre tais conhecimentos, habilidades e atitudes, o vínculo com o adolescente pode ser fragilizado (Dias \& Oliveira, 2009).

No contexto do atendimento às adolescentes gestantes, os profissionais encontram uma variedade de experiências pessoais, familiares, sociais e culturais que perpassam a situação de gravidez e projeto familiar das jovens. Isso exige dos profissionais uma postura humanizada, isenta de julgamentos e preconceitos, tipo de comportamento que pode promover a formação de relações de empatia e de vínculos entre profissional e gestante (Carvalho \& Araújo, 2007; Buendgens \& Zampieri, 2012). A segurança do atendimento e o estabelecimento de vínculo com a equipe são importantes para a humanização no atendimento em saúde, o que favorece a adesão das adolescentes às consultas (Costa, Guilherme, \& Walter, 2005). Desde a primeira consulta, a gestante busca esclarecer suas dúvidas e ter suas ansiedades amenizadas. Por esta razão, os profissionais devem estar preparados para acolhê-las, estabelecendo uma relação de confiança e respeito mútuo (Ministério da Saúde, 2005). Assim, atitudes de descaso e discriminação com as adolescentes gestantes tornam-se barreiras para a qualidade e continuidade do atendimento (Costa, Guilherme, \& Walter, 2005).

o Ministério da Saúde indica que uma parcela significativa de adolescentes grávidas busca assistência médica no Sistema Único de Saúde (SUS). 0 SUS é regido principalmente pela Política Nacional de Atenção Básica (PNAB), que indica que a equipe básica de assistência deve ser multiprofissional, contando com diferentes profissionais (médico, enfermeiro, cirurgião dentista, auxiliar de consultório dentário ou técnico em higiene dental, auxiliar de enfermagem ou técnico de enfermagem, agente comunitário, entre outros) (Ministério da Saúde, 2006). Entretanto, a equipe de atendimento em saúde à gestante, seja ela adolescente ou não, é caracterizada principalmente por profissionais da área da medicina e da enfermagem. Estes profissionais constituem a "porta de entrada" para o atendimento em saúde da gestante adolescente (Damiani, 2005).

No contexto da saúde pública brasileira, é comum que os atendimentos em saúde, assistência pré-natal e parto das gestantes ocorram em hospitais universitários. Nestes locais, os profissionais que realizam o atendimento são predominantemente alunos do curso de medicina e enfermagem que estão em formação acadêmica (Marcon, 1997; Almeida, Guinsburg, Costa, Anchieta, \& Freire, 2005). Neste contexto, considera-se que as opiniões de estudantes de medicina e enfermagem sobre o fenômeno da gravidez adolescente pode influenciar na maneira como esses profissionais irão atuar no atendimento preventivo e assistencial prestado às gestantes. De fato, as crenças que as pessoas possuem sobre determinado fenômeno influenciam no comportamento que elas adotam frente a ele. Este argumento baseia-se no modelo proposto pela Psicologia Cognitiva, que entende o comportamento humano como resultado das crenças que o indivíduo possui sobre si mesmo, seu mundo e sobre as outras pessoas (Beck, 2013).

Assim, este estudo busca conhecer as opiniões de estudantes de medicina e enfermagem sobre as causas da gravidez durante a adolescência e sobre o atendimento em saúde prestado a essa população. Este mapeamento de opiniões pode auxiliar na compreensão de valores, atitudes e pré-concepções que podem influenciar no comportamento dos profissionais, e, por consequência, na qualidade do atendimento na situação de gravidez adolescente.

\section{Método}

Participaram deste estudo 106 estudantes universitários de ambos os sexos (68 mulheres e 38 homens), com idades entre 18 e 27 anos, matriculados nos semestre iniciais dos cursos de Enfermagem e Medicina da Universidade de Passo Fundo. Destes, 63 estudantes frequentavam o $4^{\circ}$ semestre do curso de Medicina (30 alunos vinculados ao primeiro e segundo semestre e 33 alunos vinculados ao terceiro e quarto semestre do curso) e 43 estudantes frequentavam o curso de Enfermagem até o $2^{\circ}$ semestre (20 
alunos vinculados ao primeiro semestre e 23 alunos vinculados ao segundo semestre do curso).

Utilizou-se como instrumento para a coleta de informações um questionário com questões abertas, elaborado especialmente para este estudo. As questões buscaram conhecer a opinião dos estudantes sobre aspectos relacionados à ocorrência da gestação durante a adolescência e sobre o atendimento em saúde prestado a esta população. As informações coletadas foram submetidas e analisadas por meio da uma análise de conteúdo temática (Bardin, 2006).

A análise de informações na pesquisa qualitativa é um processo não matemático e de interpretação, cujo objetivo é descobrir conceitos e relações das informações brutas e de organizar esses conceitos, de forma sistemática, em um esquema explanatório teórico, (Strauss \& Corbin, 2008). Neste tipo de pesquisa é comum a utilização da técnica de análise de conteúdo temática (Gil, 2007), que consiste na adoção de uma sequência de atividades, a saber: leitura exaustiva das informações coletadas na pesquisa e categorização e interpretação dos dados obtidos. 0 tema, enquanto unidade de registro, é utilizado para estudar motivações, opiniões, atitudes, valores, crenças, tendências, etc. (Bardin, 2006). Todos os procedimentos éticos com pesquisas com seres humanos foram observados durante a realização do estudo.

\section{Resultados e discussões}

As categorias de análise foram construídas a partir das questões propostas no instrumento de coleta de informações. As questões investigaram a opinião dos estudantes sobre as causas da gravidez adolescente, as dificuldades que permeiam o atendimento à adolescente gestante, as principais dúvidas da gestante adolescente sobre sua saúde, as diferenças entre o atendimento à gestante adolescente e à adulta e as opiniões sobre quais profissionais devem fazer parte da equipe de atendimento em saúde da gestante adolescente.

\section{Causas da gravidez durante a adolescência}

Esta categoria identifica a opinião dos estudantes sobre as possíveis causas da gestação durante o período da adolescência. As razões mais citadas pelos estudantes estiveram relacionadas aos comportamentos imaturos presentes na adolescência $(46,06 \%)$. Estes comportamentos tornariam as adolescentes vulneráveis à ocorrência da gravidez precoce. Algumas respostas que ilustram esta categoria são: "irresponsabilidade", "despreocupação com a vida delas", "adolescentes não tem responsabilidade necessária", "impulsividade", "imprudência", "porque acham que nunca vai acontecer com elas (autoconfiança)", "só pensam no momento", "por acharem que uma vez só não vai engravidar" e "imaturidade". A imaturidade do adolescente se relaciona, principalmente, a algumas dificuldades em prever situações e suas possíveis relações causais (Berlofi, Alkmin, Barbieri, Guazzelli, \& Araújo, 2006; Dias \& Teixeira, 2010). Essa dificuldade pode limitar o planejamento de vida futura, principalmente no que se refere ao planejamento familiar.

Entretanto, nem sempre a ocorrência da gravidez durante a adolescência é resultado de comportamentos sexuais que revelam irresponsabilidade e imaturidade. É possível que algumas jovens, provenientes de certos contextos sociais, desejem e planejem gestar durante esse período (Dias, Jager, Patias, \& Oliveira, no prelo). Para essas jovens, o desejo de ter um filho, bem como a valorização da gravidez e da maternidade, pode associar-se às representações e valores positivos que ser mãe assume na sociedade ocidental (Lima, et al., 2004). Contudo, os estudantes não citaram a possibilidade de que a gravidez e maternidade podem representar uma opção de vida para a jovem, o que indica que os estudantes podem não estar reconhecendo a influência do contexto sociocultural das adolescentes como um dos fatores determinante na ocorrência da gestação. Esse não reconhecimento pode ser decorrente de uma formação acadêmica vinculada predominantemente ao modelo biomédico. Este modelo possui um foco sobre os processos físicos, tais como patologia e bioquímica e sobre a fisiologia do corpo humano, não levando em consideração fatores sociais e subjetivos que interferem no processo saúde-doença dos indivíduos (Guedes, Nogueira, \& Camargo Junior, 2006). Desta forma, os estudantes participantes deste estudo podem estar respondendo às expectativas atreladas ao modelo biomédico para realizar o atendimento em saúde da gestante adolescente, ou seja, os estudantes estão reproduzindo na prática a formação teórica ensinada nos cursos de graduação.

Psicol. Argum. 2014, 32(79), Supl. 2, 77-88 
A segunda causa para a ocorrência da gravidez adolescente citada pelos participantes é a desinformação sobre a utilização de métodos contraceptivos $(20,79 \%)$. Os participantes consideram importante a informação sobre a utilização correta da contracepção. Algumas respostas ilustram essa categoria: "falta de instruções nas escolas", "falta de conhecimento em contracepção", "falta de orientação educacional nas escolas", "falhas na educação", "falta de escolaridade" e "falta de orientação sobre métodos contraceptivos".

A ausência ou má utilização de métodos contraceptivos é uma das principais causas da ocorrência de gravidez entre adolescentes. Diferentes razões se associam com a não utilização ou uso esporádico de métodos anticoncepcionais, tais como dúvidas sobre a utilização correta de métodos contraceptivos, falta de informações sobre como acessá-los, medo de que os pais descubram a iniciação sexual, crença de que a pílula anticoncepcional pode engordar e sentimentos de que a não utilização de camisinha é uma prova de confiança ao seu companheiro (Patias, Jager, Fiorin, \& Dias, 2011; Neto, Dias, Rocha, \& Cunha, 2007).

De fato, 45 a $60 \%$ dos adolescentes brasileiros iniciam a vida sexual sem utilizar nenhuma medida contraceptiva (Santos \& Carvalho, 2006). Um dos motivos para a ausência ou má utilização de métodos contraceptivos por adolescentes podem derivar de problemas na transmissão de informação sobre contracepção (Patias, et al., 2011; Neto, et al., 2007). Informações parciais ou acompanhadas de preconceito podem deixar os jovens inseguros, tornando-os vulneráveis ao exercício de comportamentos sexuais de risco (Dadoorian, 2003). A influência da cultura patriarcal, a religião, a escola, bem como as questões políticas e econômicas estão fortemente presentes na formação da sexualidade. A sexualidade de adolescentes comumente é tratada de forma estritamente preventiva e punitiva. De fato, a falta de diálogo e a educação autoritário-punitiva contribuem para a existência de mitos e tabus sobre a sexualidade, colocando os adolescentes em um grupo de risco (Brêtas \& Silva, 2005).

0 terceiro fator citado pelos participantes desta pesquisa como responsável pela ocorrência da gestação adolescente é a negligência familiar (educação familiar inadequada ou falta de comunicação sobre sexualidade na família) (11,38\%). Algumas respostas que ilustram essa categoria são: "educação familiar inadequada", "descuido dos pais do adolescente para falar a respeito", "pouca informação na criação dada pelos pais", "falta de instrução familiar" e "falta de diálogo entre pais e filhos".

0 tema da sexualidade ainda encontra-se cercado de mistérios e tabus, no qual o diálogo entre pais e filhos adolescentes muitas vezes é marcado pelo silêncio. Isso faz com que os adolescentes busquem sanar suas dúvidas com outros adolescentes também inexperientes, contribuindo assim para a prática de sexo inseguro (Fernandes, Souza, \& Barroso, 2004). As conversas sobre sexualidade entre pais e filhos não são frequentes, o que contribui para a má utilização de métodos contraceptivos pelos jovens e para a ocorrência da gestação adolescente (Monteiro, Costa, Nascimento, \& Aguiar, 2007; Caputo \& Bordin, 2008). Quando essas conversas existem, elas parecem transmitir informações parciais e incompletas, em função das dificuldades dos pais em falar sobre sexualidade com seus filhos. Esses fatores indicam que a família, em certas situações, tornam as adolescentes vulneráveis a comportamentos sexuais de risco e ocorrência da gravidez (Fernandes, Junior, \& Gualda, 2012).

0 motivo menos citado pelos estudantes para explicar a ocorrência de gestação entre adolescentes está direcionado ao relacionamento amoroso da adolescente com o parceiro $(4,94 \%)$. Esses estudantes consideram que a gravidez pode ser utilizada como uma forma de manter a relação com o parceiro. Alguns exemplos de respostas que retratam esta categoria são: "para tentar segurar o companheiro", "para dar o golpe da barriga", "exigência do parceiro", "para continuar o relacionamento", "ilusões amorosas" e "para não perder o namorado/noivo".

Algumas pesquisas indicam que não existe, necessariamente, uma relação entre a ocorrência da gestação e o estabelecimento de uma união conjugal. No estudo conduzido por Moura (2003) com gestantes adolescentes e adultas jovens que realizavam acompanhamento pré-natal em serviço público de referência em Feira da Santana verificou-se que $44,1 \%$ das gestantes coabitavam com o pai da criança e $46,6 \%$, moravam com as suas famílias de origem. Em uma pesquisa multicêntrica, realizada em Porto Alegre, Rio de Janeiro e Salvador, constatou-se também que a maioria das gestações de adolescentes ocorreu na ausência da união conjugal, 74,2\% das participantes moravam com suas famílias de 
origem e apenas $15,8 \%$ das gestantes coabitavam com o parceiro (Aquino et al., 2003).

Entretanto, em um estudo realizado por Neiverth e Alves (2003), na cidade de Palotina, no Paraná, com adolescentes de 13 a 18 anos que passaram pela experiência de gestação, identificou-se que $42 \%$ das jovens foram morar com seus parceiros após a descoberta da gravidez, 29\% casaram por meio do registro civil e $29 \%$ permaneceram solteiras. Observa-se então que a união conjugal não representa, necessariamente, uma consequência da gravidez e um objetivo entre meninas que engravidam (Dias et al., no prelo).

\section{Dificuldades que permeiam 0 atendimento à gestante adolescente}

Esta categoria discute as opiniões dos estudantes sobre o atendimento à gestante adolescente $\mathrm{e}$ seus desafios. A maior dificuldade encontrada pelos profissionais se refere à responsabilização da mãe adolescente pela efetivação das orientações médicas (42,86\%). Alguns estudantes participantes do estudo consideram que as gestantes são pouco responsáveis e não apresentam adesão ao tratamento ou comprometimento com o seu acompanhamento pré-natal. Alguns exemplos de respostas confirmam esta afirmação: "conscientizar a adolescente da responsabilidade incumbida a ela", "falta de cooperação da gestante", "adesão ao tratamento", "imaturidade diante a responsabilidades", "falta de responsabilidade", "compromisso com o pré-natal" e "convencê-la dos cuidados que deve tomar durante a gestação".

No contexto da gravidez na adolescência, a responsabilização pela baixa adesão ao tratamento durante a gestação é frequentemente atribuída à adolescente. Dificilmente são levadas em consideração as dificuldades dos profissionais em estabelecer vínculos e uma relação de confiança com a adolescente gestante (Buendgens \& Zampieri, 2012). A demanda desse público nas consultas pré-natais se refere à possibilidade de receber orientações tanto sobre aspectos físicos e biológicos da gestação, quanto para os ligados ao enfretamento de conflitos emocionais decorrentes do fenômeno (Henriques, Rocha, \& Madeira, 2010; Santos, Saunders, \& Baião, 2012). Porém, é comum que os profissionais de saúde acreditem que problemas psicológicos e/ou sociais enfrentados pelos adolescentes não são de ordem médica e, portanto, não são da responsabilidade ou foco de trabalho para esses profissionais (Figueroa et al., 1991).

Ainda, alguns profissionais de saúde, em decorrência de preconceitos em relação à maternidade durante a adolescência, sentem dificuldades de reconhecer a demanda psicossocial presente neste fenômeno. Essa dificuldade pode gerar comportamentos de rejeição e aversão da adolescente gestante ao acompanhamento pré-natal. Assim, as dificuldades encontradas no atendimento à gestante também podem ser decorrentes de dificuldades particulares dos profissionais de saúde em intervir com esta população e não apenas relacionadas a comportamentos negligentes da adolescente, quando se encontram presentes. Estes fatores devem ser avaliados para determinar a etiologia de dificuldades no acompanhamento pré-natal de adolescentes grávidas (Henriques, Rocha, \& Madeira, 2010; Santos, Saunders, \& Baião, 2012).

Poucos estudantes $(16,19 \%)$ reconhecem que existem profissionais desqualificados para trabalhar e orientar pacientes e familiares em situações de gravidez precoce. Estes fragmentos das respostas ilustram esta afirmação: "preconceito dos profissionais", "falta de preparo profissional" e "má orientação dos médicos". A adesão ao pré-natal e a confiança à prescrição médica está diretamente ligada à qualidade dos atendimentos e ao estabelecimento de uma boa relação entre profissionais de saúde e adolescentes gestantes (Silva et al., 2012). Para que isto ocorra é necessário um atendimento com respeito à ética, permeado pelo cuidado às questões biopsicossociais da adolescente, sem interferência de julgamentos morais e preconceitos por parte dos profissionais (Santos, Maraschin, \& Caldeira, 2007).

Outro aspecto que dificulta o atendimento, segundo a opinião de alguns estudantes, se refere às questões psicológicas que a gestação provoca na adolescente $(23,81 \%)$. Os estudantes acreditam que a gestação é capaz de gerar sentimentos negativos na adolescente, como vergonha, ansiedade, angústia e medos. Esses sentimentos podem interferir no atendimento da jovem. Alguns fragmentos de respostas ilustram essas afirmações: "lidar com o aspecto psicológico da gestante", "mudanças emocionais que poderão ocorrer", "é insegura", "lidar com suas angústias e medos", "entenda com clareza a situação que está passando", "fazer com a adolescente amadureça" e "vergonha da situação".

Psicol. Argum. 2014, 32(79), Supl. 2, 77-88 
No meio acadêmico não é comum a valorização da compreensão de características psicoemocionais da adolescência, da sexualidade humana e suas particularidades contextuais, uma vez que o enfoque biomédico prioriza as questões físicas e biológicas (Ferrari, Thomson, \& Melchior, 2006). Desta maneira, parece difícil que os estudantes reconheçam estas questões durante $\mathrm{o}$ atendimento à gestante. Isto pode evidenciar uma falha na formação profissional em saúde, uma vez que o atendimento às adolescentes gestantes pode representar uma oportunidade para a atenção integral (saúde física, mental e social) à adolescente (Miranda \& Bouzas, 2008).

Alguns estudantes também citaram a fragilidade da rede de apoio familiar e social da gestante adolescente como um fator capaz de dificultar os atendimentos em situações de gravidez na adolescência $(15,24 \%)$. Alguns fragmentos de respostas ilustram esta opinião: "proteção dos pais", "falta de informação", "falta de apoio pedagógico" e "não procuram atendimento por esconderem dos pais". A fragilidade de rede de apoio familiar e social é realmente um fator que coloca obstáculos frente ao atendimento em saúde da gestante adolescente. No que se refere ao apoio familiar, em contextos nos quais não há aceitação a gravidez durante a adolescência, é comum que algumas adolescentes tentam negá-la e escondê-la. Este comportamento coloca em risco a atitude de prevenção de doença e promoção de saúde da adolescente em relação à sua gravidez (Pelloso, Carvalho, \& Valsecchi, 2002).

No que se refere à rede de apoio social, percebe-se que o ambiente escolar e a rede de saúde pública, no geral, não parecem estar preparados para realizar orientações e ofertar informações suficientes para atender as demandas emocionais e sociais da gestante adolescente (Ministério da Saúde, 2002). Assim, o comportamento de negação da adolescente, um ambiente familiar hostil e a fragilidade das escolas e do sistema de saúde favorecem o acesso tardio aos serviços para a realização do pré-natal; esses fatores também acabam gerando conflitos na relação do profissional de saúde com a gestante (Pelloso, Carvalho, \& Valsecchi, 2002).

\section{As principais dúvidas da gestante adolescente}

Esta categoria descreve a opinião dos participantes da pesquisa sobre as principais dúvidas que as gestantes adolescentes apresentam sobre sua gravidez e maternidade. Na opinião dos estudantes, a principal dúvida de uma gestante adolescente está relacionada aos cuidados que ela deverá ter consigo ou com o bebê $(38,49 \%)$. Os estudantes acreditam que exista uma preocupação destas jovens em desempenhar de forma positiva o seu papel materno. Fragmentos de respostas ilustram estas opiniões: "dúvidas de como cuidar do bebê", "quais os cuidados com a gravidez", "sobre amamentação", "quais os procedimento pré-natal", "como se comportar durante a gravidez", "como educar a criança" e "quais obrigações que uma mãe tem".

De fato, as preocupações das adolescentes grávidas entrevistadas no estudo de Dias et al. (no prelo) se relacionam ao desejo de cuidar e educar o filho. Preocupações semelhantes foram encontradas por Dias (2009), em um estudo realizado com jovens de diferentes regiões do país que passaram pela experiência da gestação. As preocupações das jovens mães referiam-se a "criar o filho", "dar uma boa escola e segurança para os filhos", "dar ao filho tudo que ele merece" e "garantir o futuro do filho". 0 estudo de Rodrigues, Rodrigues, Silva, Jorge e Vasconcelos (2009) também descreve preocupações equivalentes em adolescentes puérperas. Os autores demonstraram que cuidar, oferecer uma boa educação, proporcionar carinho e fornecer bons exemplos aos filhos são preocupações presentes na situação de maternidade na adolescência. Além disso, os autores perceberam que as mães adolescentes esperam saber identificar de forma adequada as necessidades de seus bebês. Essas preocupações podem decorrer da falta de experiência das adolescentes em desempenhar o papel de mãe, retratadas nas dificuldades em amamentar, identificar doenças, motivos de choro e insegurança no processo de cuidar da criança.

Independentemente de ter ou não desejado ser mãe, o papel materno se coloca para a adolescente e passa a assumir um espaço significativo na sua vida (Falcão \& Salomão, 2005). As dificuldades, as inseguranças e a falta de habilidades para o exercício do papel materno, associadas aos precários conhecimentos sobre desenvolvimento infantil que a maioria das adolescentes possui, podem configurar um quadro de risco para o desenvolvimento do bebê, uma vez que as respostas das jovens mães às demandas de seus filhos tende a ser aquém ou além das necessidades reais da criança (Bigras \& Paquette, 2007). 
Na opinião dos estudantes entrevistados, a segunda dúvida das gestantes adolescentes está relacionada à rede de apoio com quem poderão contar para enfrentar as adversidades de ser mãe precocemente $(28,87 \%)$. Os seguintes fragmentos ilustram as opiniões dos jovens: "preocupação social", "se vão deixá-la sozinha", "aceitação das pessoas", "como enfrentar o preconceito advindo da sociedade", "como retornar à escola", "oportunidades de emprego", "quanto ao seu futuro", "se o pai da criança vai me ajudar" e "como contar a família".

A rede de apoio social é um importante suporte para a adolescente gestante enfrentar as possíveis dificuldades encontradas no desempenho da maternidade. Entendem-se como apoio social os recursos relacionais que uma pessoa dispõe para enfrentar diferentes situações na vida que geram estresse. Este conceito engloba o número de pessoas com as quais o sujeito se relaciona, a estrutura e a qualidade destas relações (Moreira \& Sarriera, 2008). Esse apoio pode ser de caráter emocional, socioemocional e/ou instrumental. 0 apoio emocional é caracterizado pelo apoio à autoestima, retratado pela valorização positiva de si mesmo e no respeito e no acolhimento da condição de adolescente, mulher e mãe. 0 apoio socioemocional se refere ao afeto, à compreensão e à aceitação por parte dos outros. 0 apoio instrumental, por sua vez, é caracterizado pela oferta de informações, auxílio econômico, ajuda e orientações para enfrentar situações difíceis (Estramiana, 1992). Essas formas de apoio funcionam como moderadoras dos sentimentos negativos que podem emergir com a gravidez. Nesse sentido, é importante que a adolescente receba esses três tipos de apoio, uma vez que estes podem auxiliar na promoção de saúde tanto durante a gestação como depois do parto (Moreira \& Sarriera, 2008).

0 apoio social pode ser oferecido pela família, pelo companheiro, pela escola e pela sociedade no geral. A família é uma rede de apoio importante no contexto da gravidez adolescente e exerce um papel significativo na oferta de apoio social. É comum que a família auxilie no cumprimento de responsabilidades e acúmulo de tarefas que a adolescente poderá enfrentar. Com o nascimento do bebê, a adolescente passa a somar em suas atividades diárias os cuidados com a criança. 0 apoio social por parte dos pais parece ser essencial tanto no plano financeiro quanto na orientação com os cuidados ao bebê (Zagury, 1996).
Os estudantes ainda reconhecem que as jovens apresentam algumas preocupações relacionadas às mudanças fisiológicas decorrentes da gestação $(27,2 \%)$. A seguir são apresentados fragmentos de respostas que ilustram esta afirmação: "quais as modificações fisiológicas", "quais as mudança no corpo", "que tipo de opção de parto", "como deve ser a alimentação" e "quais exercícios físicos recomendados".

A gravidez é um período de transformações para a mulher, seja ela adolescente ou não. Seu corpo se modifica e seus níveis de hormônios se alteram para a manutenção do feto. Neste período são comuns temores sobre alterações na autoimagem corporal e preocupações relacionadas a não ter uma criança saudável. Outros temores estão relacionados ao feto e à função de gerar, nutrir e dar a luz (Moreira, Viana, Queiroz, \& Jorge, 2008). No que se refere ao o parto, destaca-se que este é um momento crítico, uma vez que se dá a passagem de um estado a outro: do bebê na barriga para o bebê nos braços. Outra situação que aumenta a ansiedade e a insegurança é a incapacidade de saber como e quando vai ocorrer o trabalho de parto, isto é, a impossibilidade de controlar o processo. 0 parto se torna um momento imprevisível e desconhecido (Maldonado, 2002).

\section{Profissionais necessários ao atendimento da gestante adolescente}

Esta categoria descreve a opinião dos estudantes sobre quem são os profissionais que devem integrar a equipe de atendimento a saúde das adolescentes gestantes. Entre os profissionais mais citados pelos estudantes estão Médico ou ginecologista (28,57\%), Psicólogo (27,89\%), Enfermeiro $(14,97 \%)$ e Assistente Social $(6,46 \%)$. As demais profissionais de saúde (Psiquiatra, Fisioterapeuta, Técnicos em Enfermagem) não obtiveram 3\% das respostas dos participantes.

A opinião apresentada pelos estudantes diverge da opinião de médicos e enfermeiros entrevistados no estudo sobre o mesmo assunto, realizado por Dias e Oliveira (2009). Os profissionais de saúde identificam que a equipe multidisciplinar deve ser formada por um médico com especialidade na adolescência, psiquiatra, psicólogo, assistente social, nutricionista, recreacionista, enfermeiro e um coordenador para integras os diferentes profissionais. Independentemente de quais profissionais que integram a equipe de atendimento, é necessário que

Psicol. Argum. 2014, 32(79), Supl. 2, 77-88 
fique claro que o trabalho com adolescentes é um desafio para os profissionais de saúde. Trabalhar com adolescentes exige dos profissionais um olhar humanizado e o reconhecimento da adolescência como uma vivência como ambivalente, diferenciada e, na maioria das vezes, geradora de conflitos (Moreira et al., 2008).

0 atendimento multidisciplinar ao adolescente é importante porque em equipe o profissional pode buscar suprir suas deficiências por meio da supervisão e da troca de experiências. Este momento de troca pode envolver não somente aspectos técnicos, como também aspectos afetivos. Com o apoio da equipe, os profissionais que se sentem despreparados podem sentir mais segurança na hora de acolher e atender um adolescente com problemas de saúde ou em situação de gravidez. Neste sentido, é necessário superar o conhecimento compartimentado e fragmentado do ser humano, buscando compreendê-lo em sua totalidade e atendê-lo levando em consideração não apenas o seu estado de saúde, mas o indivíduo em desenvolvimento (Dias \& Oliveira, 2009).

Além do médico, um percentual significativo de respostas dos estudantes sugere a importância do psicólogo no atendimento a esta população $(27,89 \%)$. A atuação do psicólogo no ambiente de atendimento à saúde busca o alívio emocional do paciente e de sua família. No contexto do acompanhamento pré-natal e período do parto, o trabalho do psicólogo é importante, uma vez que é comum a emergência de diversos aspectos psicoemocionais relacionados à transição para a parentalidade. A escuta qualificada pode auxiliar nesses momentos específicos na vida da mulher e do casal. Entretanto, ainda existem poucas ações sendo efetivamente realizadas para que existam estes espaços de escuta diferenciada e apoio emocional durante o acompanhamento pré-natal e parto (Rodrigues \& Siqueira, 2008).

Os principais profissionais comumente envolvidos na assistência pré-natal são médicos e enfermeiras, que muitas vezes não têm em sua formação o treinamento e preparo para realizar uma escuta diferenciada, voltando o atendimento para os aspectos técnicos e orientações básicas (Nagahama \& Santiago, 2008). Nesse sentido, a participação de psicólogos no pré-natal pode ser de grande valia. Contudo, apesar da contribuição que este profissional pode trazer no período gestacional, preparação para o parto e puerpério, sua atuação e efetiva inserção ainda são escassas (Saraiva, 2007). Somado a isso, no que tange a preparação para o parto, Dias e Deslandes (2006) sustentam que a hora do nascimento é um momento em que a maioria das mulheres precisa tanto do suporte psicoafetivo quanto de intervenções médicas. Essas questões tornam-se mais evidentes especialmente para a gestante adolescente devido a todos os aspectos emocionais características a esta fase da vida, que quando associados a uma gravidez precoce podem tornar-se ainda mais acentuados.

\section{Considerações finais}

Este estudo buscou conhecer as opiniões de estudantes de medicina e enfermagem sobre as causas da gravidez durante a adolescência e sobre o atendimento em saúde prestado a essa população. Para os participantes da pesquisa, os motivos que levam a adolescente a gestar estariam relacionados aos comportamentos que indicam a imaturidade característica da adolescência, desinformação sobre contracepção, negligência familiar e a busca por uma estabilidade no relacionamento amoroso. No que se refere ao atendimento à gestante adolescente, os participantes descrevem algumas dificuldades e desafios, tais como negligência da mãe adolescente frente às orientações médicas, desqualificação profissional para o atendimento desta população, entre outras. Para os estudantes entrevistados, as principais dúvidas que uma gestante adolescente apresenta se referem à cuidados/responsabilidades decorrentes da maternidade, a presença de uma rede de apoio e dúvidas sobre mudanças fisiológicas. Por fim, no que diz respeito à opinião dos estudantes sobre os profissionais que devem integrar a equipe de atendimento à gestante adolescente estão, respectivamente, psicólogo, médico, enfermeiro, assistente social e o ginecologista.

Estes resultados indicam que os estudantes de medicina e enfermagem reconhecem as peculiaridades presente no atendimento da adolescente gestante e importância de um atendimento interdisciplinar. Entretanto, os participantes deste estudo não reconhecem, de fato, a influência do contexto sociocultural sobre a experiência da gestação e maternidade adolescente, apresentando crenças 
estereotipadas em relação a estas experiências. A desconsideração de varáveis socioculturais no contexto da gravidez adolescente pode ser decorrente do modelo biomédico utilizado como referência teórico-prática predominante em cursos de enfermagem e, especialmente, de medicina. Este modelo desconsidera a influência de aspectos sociais e culturais sobre o processo de saúde - doença (Guedes, Nogueira, \& Camargo Junior, 2006). Os estudantes parecem reproduzir em suas falas e práticas os pressupostos biomédicos ensinados durante a formação acadêmica.

Este estudo investigou uma amostra pequena de estudantes matriculados em apenas dois cursos da área da saúde: medicina e enfermagem, o que limita a generalização dos resultados encontrados neste estudo para uma população mais abrangente. Ainda, o instrumento de pesquisa utilizado não permitiu a investigação detalhada das crenças dos estudantes sobre a gravidez adolescente. Sugerem-se estudos com amostras maiores e que sejam capazes de representar uma população de estudantes de diferentes áreas do conhecimento em saúde. Somado a isto, estudos que utilizem métodos combinados e/ ou busquem realizar a comparação entre as opiniões de estudantes de medicina e enfermagem sobre variáveis que perpassam a situação de gravidez adolescente podem indicar como este fenômeno é percebido durante a formação acadêmica e como esta formação pode determinar práticas profissionais futuras. Este estudo não realizou a comparação entre as opiniões dos estudantes dos dois grupos, não oferecendo suporte para uma análise comparativa. Contudo, pode-se perceber similaridade entre as respostas, indicando a fragilidade na formação acadêmica em cursos de medicina e enfermagem, pelo menos no que se referem às particularidades que perpassam o atendimento em saúde da gestante adolescente.

\section{Referências}

Aquino, E. M. L., Heilborn, M. L., Knauth, D., Bozon, M., Almeida, M. C., Araújo, J., \& Menezes, $\quad$ G. (2003). Adolescência e reprodução no Brasil: a heterogeneidade dos perfis sociais. Caderno de Saúde Coletiva, 19(Sup. 2), 377-388. Retirado de: http://www.scielo. br/pdf/csp/v19s2/a19v19s2.pdf. Acesso em: 16 de novembro de 2012 .
Almeida, M.F., Guinsburg, R., Costa, J.O., Anchieta, L.M., \& Freire, L.M.S. (2005). Ensino da reanimação neonatal em maternidades públicas das capitais brasileiras. Jornal de Pediatria, 81(3), 233-239. Retirado de: http://www.scielo.br/scielo.php?pid=S0021$-75572005000400010 \&$ script=sci_arttext\&tlng=pt. Acesso em: 02 de março de 2013.

Bardin, L. (2006). Análise de Conteúdo. Lisboa: Edições 70 .

Beck, J. S. (2013). Introdução à terapia cognitivo comportamental. In J. S. Beck. Terapia cognitivo-comportamental: teoria e prática. (pp. 21-36). Porto Alegre: Artmed.

Berlofi, L. M., Alkmin, E. L. C, Barbieri, M., Guazzelli, C. A. F., \& Araújo, F. F. (2006). Prevenção da reincidência de gravidez em adolescentes: efeitos de um Programa de Planejamento Familiar. Acta Paulistana de Enfermagem, 19(2), 196-200. Retirado de: http:// www.scielo.br/scielo.php?script=sci_arttext\&pid =S0103-21002006000200011. Acesso em 13 de março de 2013.

Bigras, M. \& Paquette, D. (2007). Estudo pessoa-processo-contexto da qualidade das interações entre mãe adolescente e seu bebê. Ciência \& Saúde Coletiva, 12(5), 1167-1174. Retirado de: http://www.scielo.br/scielo.php?pid=S1413$-81232007000500013 \&$ script=sci_arttext. Acesso em 12 de abril de 2013.

Brêtas, J. R. S. \& Silva, C. V. (2005). Orientação sexual para adolescentes: relato de experiência. Acta Paulistana de Enfermagem, 18(3), 326-333. Retirado de: http:// www.scielo.br/scielo.php?script=sci_arttext\&pid =S0103-21002005000300015. Acesso em 11 de abril de 2013

Buendgens, B. B. \& Zampieri, M. F. M. (2012). A adolescente grávida na percepção de médicos e enfermeiros da atenção básica. Escola Anna Nery, 16(1), 64-72. Retirado de: http://www.scielo.br/scielo. php?pid=S1414-81452012000100009\&script=sci arttext. Acesso em 14 de novembro de 2012.

Caputo, V. G. \& Bordin, I. A. (2008). Gravidez na adolescência e uso frequente de álcool e drogas no contexto familiar. Revista de Saúde Pública, 42(3), 402-410. Retirado de: http://www.scielo.br/scielo. php?pid=S0034-89102008000300003\&script=sci arttext. Acesso em 14 de novembro de 2012.

Psicol. Argum. 2014, 32(79), Supl. 2, 77-88 
Carvalho, V. C. P. \& Araújo, T. V. B. (2007). Adequação da assistência pré-natal em gestantes atendidas em dois hospitais de referência para gravidez de alto risco do Sistema Único de Saúde, na cidade de Recife, Estado de Pernambuco. Revista Brasileira de Saúde Materno-Infantil, 7(3), 309-317. Retirado de: http://www.scielo.br/scielo.php?pid=S1519$-38292007000300010 \&$ script=sci_arttext. Acesso em 14 de novembro de 2012.

Costa, A. M., Guilherme, D., \& Walter, M. I. M. T. (2005). Atendimento a gestantes no Sistema Único de Saúde. Revista de Saúde Pública, 39(5), 768-774. Retirado de: http://www.scielo.br/scielo.php?pid=S0034$-89102005000500011 \&$ script=sci_arttext. Acesso em 16 de novembro de 2012.

Dadoorian, D. (2003). Gravidez na adolescência: um novo olhar. Psicologia Ciência \& Profissão, 23(1), 84-91. Retirado de: http://www.scielo.br/scielo. php?pid=S1414-98932003000100012\&script $=$ sci arttext. Acesso em 04 de março de 2013.

Damiani, F. E. (2005). Gravidez na adolescência: a quem cabe educar? Passo Fundo: UPF.

Dias, A. C. G. \& Teixeira, M. A. P. (2010). Gravidez na adolescência: um olhar sobre um fenômeno complexo. Paidéia, 45(20), 123-131. Retirado de: http://www.scielo.br/scielo.php?pid=S0103$-863 X 2010000100015 \&$ script=sci_arttext. Acesso em 05 de abril de 2013.

Dias, A. C. G. (2009). Análise das expectativas de jovens que vivenciaram a gravidez na juventude. In R. M. C. Libório, \& S. H. Koller. (Eds.). Adolescência e juventude: risco e proteção na realidade brasileira. (pp. 119140). Porto Alegre: Casa do Psicólogo.

Dias, A. C. G. \& Oliveira, V. Z. (2009). A percepção dos profissionais de saúde acerca do atendimento prestado ao adolescente. In A. C. G. Dias. (Eds.) Psicologia \& Saúde: pesquisas e reflexões. (pp. 63-91). Santa Maria: Editora UFSM.

Dias, A. C. G., Jager, M. E., Patias, N. D., \& Oliveira. C. T. (no prelo). Maternidade e casamento: o que pensam as adolescentes? Interacções.

Dias, M. A. B. \& Deslandes, S. F. (2006). Expectativas sobre a assistência ao parto de mulheres usuárias de uma maternidade pública do Rio de Janeiro, Brasil: os desafios de uma política de humanização da assistência. Cadernos de Saúde Pública, 22(12), 2647 2655. Retirado de: http://www.scielo.br/pdf/csp/ v22n12/13.pdf. Acesso em 15 de abril de 2013.
Estramiana, J. L. A. (1992). Desempleo y bienestar psicológico. Madrid: Siglo XXI de España.

Falcão, D. V. \& Salomão, N. M. R. (2005). 0 papel dos avós na maternidadeadolescente.Estudos de Psicologia,22(2), 205-212. Retirado de: http://www.scielo.br/scielo. php?pid=S0103-166X2005000200010\&script=sci abstract\&tlng=pt. Acesso em 15 de abril de 2013.

Fernandes, A. O., Júnior, H. P. O. S., \& Gualda, D. M. R. (2012). Gravidez na adolescência: percepções das mães de gestantes jovens. Acta Paulista de Enfermagem, 25(1), 55-60. Retirado de: http://www.scielo.br/scielo. php?pid=S0103-21002012000100010\&script=sci arttext. Acesso em 18 de abril de 2013.

Fernandes, J. F. P., Sousa, L. B., \& Barroso, M. G. T. (2004). Repercussão da gravidez no contexto sócio-familiar da adolescente - uma experiência. Acta Paulistana Enfermagem, 17(4), 400-406. Retirado de: http:// www.unifesp.br/acta/sum.php?volume=17\&numer o=4\&item=res5.htm. Acesso em 13 de abril de 2013.

Ferrari, R. A. P., Thomson, Z., \& Melchior, R. (2006). Atenção à saúde dos adolescentes: percepção dos médicos e enfermeiros das equipes da saúde da família. Cadernos de Saúde Pública 22(11), 2491-2495. Retirado de: http://www.scielosp.org/pdf/csp/ v22n11/24.pdf. Acesso em 15 de abril de 2013.

Gil, A. C. (2007). Métodos e técnicas de pesquisa social. 5. ed. São Paulo: Atlas.

Guedes, C.R., Nogueira, M.I., \& Camargo Júnior. (2006). A subjetividade como anomalia: contribuições epistemológicas para a crítica do modelo biomédico. Ciência \& Saúde Coletiva, 11(40), 1093-1103. Retirado de: http://www.scielo.br/pdf/csc/v11n4/32345.pdf. Acesso em 18 de maio de 2013.

Figueroa, E., Kolasa, K. M., Horner, R. E., Murphy, M., Dent, M. F., Ausherman, J. A., \& Irons, T. G. (1991). Attitudes, knowledge and training of medical residents regarding adolescent health issues. Journal of Adolescent Health Care, 12(6), 443-449. Retirado de: http:// www.ncbi.nlm.nih.gov/pubmed/1768697. Acesso em 18 de maio de 2013.

Henriques, B. D., Rocha, R. L., \& Madeira, A. M. F. (2010). Saúde do adolescente: o significado do atendimento para os profissionais da atenção primária do município de Viçosa, MG. Revista Médica de Minas Gerais, 20(3), 300-309. Retirado de: http://rmmg. medicina.ufmg.br/index.php/rmmg/article/viewFile/268/251. Acesso em 15 de maio de 2013. 
Lima, C. T. B., Feliciano, K. V. O., Carvalho, M. S. F., Souza, A. P. P., Manebó, J. B. C., Ramos, L. S., Cassundé, L. F., \& Kovacs, M. H. (2004). Percepções e práticas de adolescentes grávidas e de familiares em relação à gestação. Revista Brasileira de Saúde Materno-Infantil, 6(1), 71-83. Retirado de: http://www.scielo.br/pdf/rbsmi/ v4n1/19983.pdf. Acesso em 12 de dezembro de 2012.

Maldonado, M. T. Psicologia da gravidez: parto e puerpério. 16. ed. São Paulo: Saraiva, 2002.

Marcon, S.S. (1997). "Flashes" de como as gestantes percebem a assistência pré-natal em um hospital universitário. Revista Latino Americana de Enfermagem, 5(4), 4354. Retirado de: http://www.scielo.br/pdf/rlae/v5n4/ v5n4a06.pdf. Acesso em 22 de novembro de 2012.

Ministério da Saúde. (2002). Secretaria de Políticas de Saúde. Área Técnica de Saúde da Mulher. Assistência em planejamento familiar: manual técnico/secretaria de políticas de saúde. 4. ed. Brasília: Ministério da Saúde. Retirado de: http://bvsms.saude.gov.br/bvs/ publicacoes/0102assistencia1.pdf. Acesso em 15 de maio de 2013.

Ministério da Saúde. (2005). Secretaria de Atenção à Saúde. Departamento de Ações Programáticas Estratégicas. Pré-natal e puerpério: atenção qualificada e humanizada - manual técnico. Brasília: Ministério da Saúde. Retirado de: http://portal. saude.gov.br/portal/arquivos/pdf/manual_puerperio_2006.pdf. Acesso em 15 de maio de 2013.

Ministério da Saúde. (2006). Secretaria de Atenção à Saúde. Departamento de Atenção Básica. Política Nacional de Atenção Básica. 4. ed. Brasília: Ministério da Saúde, 2006. Retirado de: http://bvsms.saude. gov.br/bvs/publicacoes/politica_nacional_atencao_ basica_2006.pdf. Acesso em 18 de maio de 2013.

Miranda, A. T. C. \& Bouzas, I. C. S. (2008). Gravidez. In: Ministério da Saúde. Departamento de Ações Programáticas Estratégicas. Saúde do adolescente: competências e habilidades. (pp. 242-256). Brasília, Série B, Textos Básicos da Saúde.

Monteiro, C. F. S.; Costa, N. S. S., Nascimento, P. S. V., \& Aguiar, Y. A. (2007). A violência intrafamiliar contra adolescentes grávidas. Revista Brasileira de Enfermagem, 60(4), 373-376. Retirado de: http:// www.scielo.br/scielo.php?script=sci_arttext\&pid =S0034-71672007000400002. Acesso em 12 de setembro de 2012.
Moreira, M. C. \& Sarriera, J. C. (2008). Satisfação e composição da rede de apoio social a gestantes adolescentes. Psicologia em Estudo, 13(4), 81-789. Retirado de: http://www.scielo.br/scielo.php?script=sci_artte xt\&pid=S1413-73722008000400016. Acesso em 14 de junho de 2012.

Moreira, T. M. M., Viana, D. S., Queiroz, M. V. O., \& Jorge, M. S. B. (2008). Conflitos vivenciados pelas adolescentes com a descoberta da gravidez. Revista da Escola de Enfermagem USP, 42(2), 2008. Retirado de: http:// www.scielo.br/scielo.php?script=sci_arttext\&pid =S0080-62342008000200015. Acesso em 12 de janeiro de 2013.

Moura, M. S. Q. (2003). Características clínicas e nutricionais de gestantes e adolescentes e adultas jovens acompanhadas no pré-natal: Hospital Geral Clériston Andrade. Dissertação de Mestrado, Universidade Federal de Feira de Santana, Feira de Santana, BA.

Nagahama, E. E. I \& Santiago, S. M. (2008). Práticas de atenção ao parto e os desafios para a humanização do cuidado em dois hospitais vinculados ao SUS em município da região Sul do Brasil. Cadernos de Saúde Pública, 24(8), 1859-1868. Retirado de: http://www.scielosp.org/scielo.php?script=sci_ arttext\&pid=S0102-311X2008000800014. Acesso em 12 de setembro de 2012.

Neiverth, I. S. \& Alves, G. B. (2003). Gravidez na adolescência e mudança do papel social da mulher. Paidéia, 12(24), 229-240. Retirado de: http://www. scielo.br/scielo.php?script=sci_arttext\&pid=S0103-863X2002000300011. Acesso em 15 de fevereiro de 2013.

Neto, F. R. G. X., Dias, M. S. A., Rocha, J., \& Cunha, I. C. K. O. (2007). Gravidez na adolescência: motivos e percepções das adolescentes. Revista Brasileira de Enfermagem, 60(3), 279-285. Retirado de: http:// www.scielo.br/scielo.php?script=sci_arttext\&pid $=$ S0034-71672007000300006. Acesso em 18 de fevereiro de 2013.

Patias, N. D. Jager, M. E., Fiorin, P. C. \& Dias, A. C. G. (2011). Construção histórico social da adolescência: implicações na percepção da gravidez na adolescência como um problema. Revista Contexto \& Saúde, 10(20), 205214. Retirado de: http://www1.unijui.edu.br/revistas/index.php/revista-contexto-e-saude-edicao-atual. Acesso em 18 de fevereiro de 2013.

Psicol. Argum. 2014, 32(79), Supl. 2, 77-88 
Pelloso, S. M., Carvalho, M. D. B., \& Valsecchi, E. A. S. S. (2002). O vivenciar da gravidez na adolescência. Acta Scientiarum: Health Scienses, 24(3), 775- 781. Retirado de: http://webcache.googleusercontent.com/search?q=cache:Ln4Db4NojSAJ: eduem.uem.br/ojs/index.php/ActaSciHealthSci/ article/download $/ 2508 / 1677+\& \mathrm{~cd}=1 \& \mathrm{hl}=$ pt -BR\&ct=clnk\&gl=br. Acesso em 15 de maio de 2013.

Rodrigues, A. V. \& Siqueira, A. A. F. (2008). Sobre as dores e temores do parto: dimensões de uma escuta. Revista Brasileira de Saúde Materno-Infantil, 8(2), 179-186. Retirado de: http://www.scielo.br/scielo. php?pid=S1519-38292008000200005\&script $=$ sci_ arttext. Acesso em 12 de agosto de 2012.

Rodrigues, D. P., Rodrigues, F. R. A., Silva, L. M. S., Jorge, M. S. B., \& Vasconcelos, L. D. G. P. (2009). O adolescer e ser mãe: representações sociais de puérperas adolescentes. Cogitare Enfermagem, 4(3), 455-462. Retirado de: http://webcache.googleusercontent.com/search?q=cache:laqY9QC32pgJ:ojs. c3sl.ufpr.br/ojs 2/index.php/cogitare/arti cle/download /16166/10687+\&cd=1\&hl=pt-BR\&ct=clnk\&gl=br. Acesso em 12 de maio de 2013.

Santos, A. \& Carvalho, C. (2006). Gravidez na adolescência: um estudo exploratório. Boletim de Psicologia, 57(125), 135-151. Retirado de: http:// pepsic.bvsalud.org/scielo.php?script=sci_arttext\&p $\mathrm{id}=$ S0006-59432006000200002. Acesso em $15 \mathrm{de}$ abril de 2013.

Santos, D. R., Maraschin, M. S., \& Caldeira, S. (2007). Percepção dos enfermeiros frente à gravidez na adolescência. Ciência, Cuidado e Saúde, 6(4), 479485. Retirado de: http://webcache.googleusercontent.com/search? q=cache:IA3Zr13TEysJ:e duem.uem.br/ojs/index.php/CiencCuidSaude/ article/download/3684/2686+\&cd=1\&hl=pt-BR\&ct=clnk\&gl=br. Acesso em 12 de setembro de 2012.
Santos, M. M. A. S., Saunders, C., \& Baião, M. R. (2012). A relação interpessoal entre profissionais de saúde e adolescente gestante: distanciamentos e aproximações de uma prática integral e humanizada. Ciência \& Saúde Coletiva, 17(3), 775-786. Retirado de: http://www.scielo.br/scielo.php?pid=S1413$-81232012000300025 \&$ script=sci_arttext. Acesso em 12 de setembro de 2012.

Saraiva, S. (2007). Nascer, viver, morrer... Uma visão transcendental. In F. F. Bortoletti., A. F., Moron, J. B., Filho, M. U., Nakamura, R. M., Santana, \& R., Mattar. (Eds.). Psicologia na prática obstétrica: abordagem interdisciplinar. (pp. 5-11) Barueri, SP: Manole.

Strauss, A., \& Corbin, J. (2008). Pesquisa qualitativa: técnicas e procedimentos para o desenvolvimento da teoria fundamentada. (L.O. Rocha, Trad., 2. ed.). Porto Alegre: Artes Médicas.

Silva, R. M., Costa, M. S., Matsue, R. Y., Sousa, G. S., Catrib, A. M. F., \& Vieira, L. J. E. S. (2012). Cartografia do cuidado na saúde da gestante. Ciência \& Saúde Coletiva, 17(3), 635-642. Retirado de: http://www.scielo.br/ pdf/csc/v17n3/v17n3a09.pdf. Acesso em 15 de abril de 2013.

Zagury, T. (1996). O adolescente por ele mesmo. Rio de Janeiro: Record. 